\title{
Catalysis-in-a-Box: Robotic Screening of Catalytic Materials in the Time of COVID-19
}

\author{
Gaurav Kumar ${ }^{1, \uparrow}$, Hannah Bossert ${ }^{1, \uparrow}$, Dan McDonald ${ }^{1}$, Anargyros \\ Chatzidimitriou ${ }^{1}$, M. Alexander Ardagh ${ }^{1,5}$, Yutong Pang ${ }^{1}$, ChoongSze Lee ${ }^{1,5}$, \\ Michael Tsapatsis ${ }^{3,4,5}$, Omar A. Abdelrahman ${ }^{2,5}$, Paul J. Dauenhauer ${ }^{1,5^{\star}}$ \\ ${ }^{1}$ University of Minnesota, Department of Chemical Engineering and Materials Science, 421 Washington Ave. SE, \\ Minneapolis, MN 55455 USA \\ ${ }^{2}$ Department of Chemical Engineering, University of Massachusetts Amherst, 686 N. Pleasant Street, Amherst, MA \\ 01003 USA \\ ${ }^{3}$ Department of Chemical and Biomolecular Engineering \& Institute for NanoBioTechnology, Johns Hopkins \\ University, 3400 N. Charles Street, Baltimore, MD 21218 USA \\ ${ }^{4}$ Johns Hopkins University, Applied Physics Laboratory, 11100 Johns Hopkins Road, Laurel, MD 20723 USA \\ ${ }^{5}$ Catalysis Center for Energy Innovation, University of Delaware, 150 Academy Street, Newark, Delaware 19716 \\ $\dagger$ Authors contributed equally. \\ * Corresponding author: hauer@umn.edu
}

\begin{abstract}
The emergence of a viral pandemic has motivated the transition away from traditional, laborintensive materials testing techniques to new automated approaches without compromising on data quality and at costs viable for academic laboratories. Reported here is the design and implementation of an autonomous micro-flow reactor for catalyst evaluation condensing conventional laboratory-scale analogues within a single gas chromatograph (GC), enabling the control of relevant parameters including reactor temperature and reactant partial pressures directly from the GC. Inquiries into the hydrodynamic behavior, temperature control, and heat/mass transfer were sought to evaluate the efficacy of the microflow reactor for kinetic measurements. As a catalyst material screening example, a combination of four Brønsted acid catalyzed probe reactions, namely the dehydration of ethanol, 2-propanol, 1-butanol, and the dehydra-decyclization of 2-methyltetrahydrofuran on a solid acid HZSM-5 (Si/Al 140), were carried out in the temperature range 403-543 K for the measurement of apparent reaction kinetics. Product selectivities, proton-normalized reaction rates, and apparent activation barriers were in agreement with measurements performed on conventional packed bed flow reactors. Furthermore, the developed microflow reactor was demonstrated to be about ten-fold cheaper to fabricate than commercial automated laboratory-scale reactor setups and is intended to be used for kinetic investigations in vapor-phase catalytic chemistries, with the key benefits including automation, low cost, and limited experimental equipment instrumentation.
\end{abstract}

1.0 Introduction. The rapid onset of coronavirus disease 2019 (COVID-19) as a dangerous infection spreadable by people in close contact has transformed the nature of materials and chemical research. $^{1,2}$ While the past two decades have produced a wave of new catalyst materials including hierarchical zeolites, ${ }^{3}$ MOFs, ${ }^{4}$ singleatom surfaces, ${ }^{5}$ intermetallic structures, ${ }^{6}$ and other low-dimensional materials ${ }^{7}$, testing these catalysts to determine structure-performance relationships requires researchers to work in close proximity. Such in-person catalytic performance evaluation tends to be a multi-step process, where the traditional approaches have involved qualitative screening techniques ${ }^{8-12}$ in search for the 'best' catalyst for the envisioned application, followed by kinetic interrogations of the shortlisted catalysts to establish reactivity, selectivity, and stability trends and subsequent detailed mechanistic inquiries into reaction pathways. ${ }^{9,12}$ To continue this effective general approach to assessing materials performance, laboratory operations must transition to more automated methods requiring only minimal manual intervention. 
The challenge of transitioning laboratories to more robotic operations arises from the complexity of catalytic materials testing. In particular, kinetic studies frequently utilize differential reactors to measure either initial or steady state rates of reaction over a wide range of operating conditions. For reactions occurring on gas-solid interfaces, such measurements are typically performed in packed bed flow reactors (PBRs), ${ }^{12-17}$ which remain the workhorse of any heterogeneous catalysis laboratory. Importantly, laboratory-scale costeffective PBRs are often custom-fabricated, and they require constant human monitoring owing to multiple independent process control elements, and separate, often non-coupled, reaction and separation/quantification components. ${ }^{9,12}$ The manual interventions on these equipment typically involve operations like switching valves, and/or precisely altering reaction conditions at determined intervals. Furthermore, data analysis from common analytical instruments on these PBRs (like gas chromatography units (GCs)) remains largely manual. Together, these factors lead to cumbersome experimentation and analysis, especially in scenarios requiring kinetic measurements for many catalysts or assessing catalyst stability on-stream for prolonged periods.

The ability to perform high fidelity kinetic studies with minimal human oversight would greatly reduce the labor and costs associated with catalyst development and discovery, and recent research efforts reflect this interest. ${ }^{18}$ Flow reactors ${ }^{19-21}$ as well as control algorithms ${ }^{22-25}$ are increasingly integrated with online analytical tools to collect and analyze kinetic data without user supervision. However, these experimental systems are almost always fabricated for a specific targeted application, and important heat and mass transfer considerations are often not explicitly reported, dissuading other research groups to invest time and resources to fabricate them. One straightforward implementation of low-cost automated microreactors with broad applicability among previously reported approaches is the pulsed-flow technique, which involves dosing a pulse of reactant over a catalytic material to study product distribution trends. This approach is readily integrated within a commercial gas chromatograph (GC), ${ }^{26-32}$ but the transient nature of the technique limits kinetic parameter estimation. In addition, the product distributions under such transient conditions may vary from corresponding steadystate values. ${ }^{16}$ Extending this approach to modify existing GCs, enabling reliable but largely automated kinetics measurements in a continuous flow method while also facilitating safe laboratory operation has been the focus of this report.

Here, the automated vapor-phase continuous micro-flow reactor is described as integrated within a gas chromatograph (GC) unit capable of measuring the reaction kinetics of vapor-phase catalytic chemistries with volatile feeds (either gases or vaporizable liquids). The design and implementation of the setup is first presented. This is followed by detailed investigation of heat and mass transfer characteristics under cold-flow as well as reaction conditions by a recently developed online toolbox (GradientCheck) reported by Ribeiro and co-workers. ${ }^{33}$ Thereafter, kinetic parameters (namely turnover rates, apparent activation barriers, and product distributions) for acid-catalysed vapor-phase dehydration of three alcohols (ethanol, 2-propanol, 1-butanol) and a cyclic ether (2-methyltetrahydrofuran) on a solid acid catalyst (HZSM-5, Si/Al 140) are measured and compared with previously reported values under similar conditions. To address the laborintensive nature of chromatogram data handling, a chromatogram analysis tool is developed in-house and integrated to the experimentation workflow for automatic GC peak detection and peak area quantification.

The fabricated setup as well as the reported data analysis tool are primarily intended for materials researchers to obtain kinetic data of vapor-phase chemistries with minimal user-supervision and safe working conditions. The provided information along with the detailed analysis of the performance of the device enable simple implementation in any laboratory that is testing catalytic materials. Extensive supporting information provides detailed parts lists and instructions for implementation with both hardware and software modifications. Additionally, the provided analysis means that immediate transition of a laboratory to safer, more robotic catalyst materials testing can be conducted with the justification that resulting data achieves the quality obtained using conventional methods. 


\section{Top view}
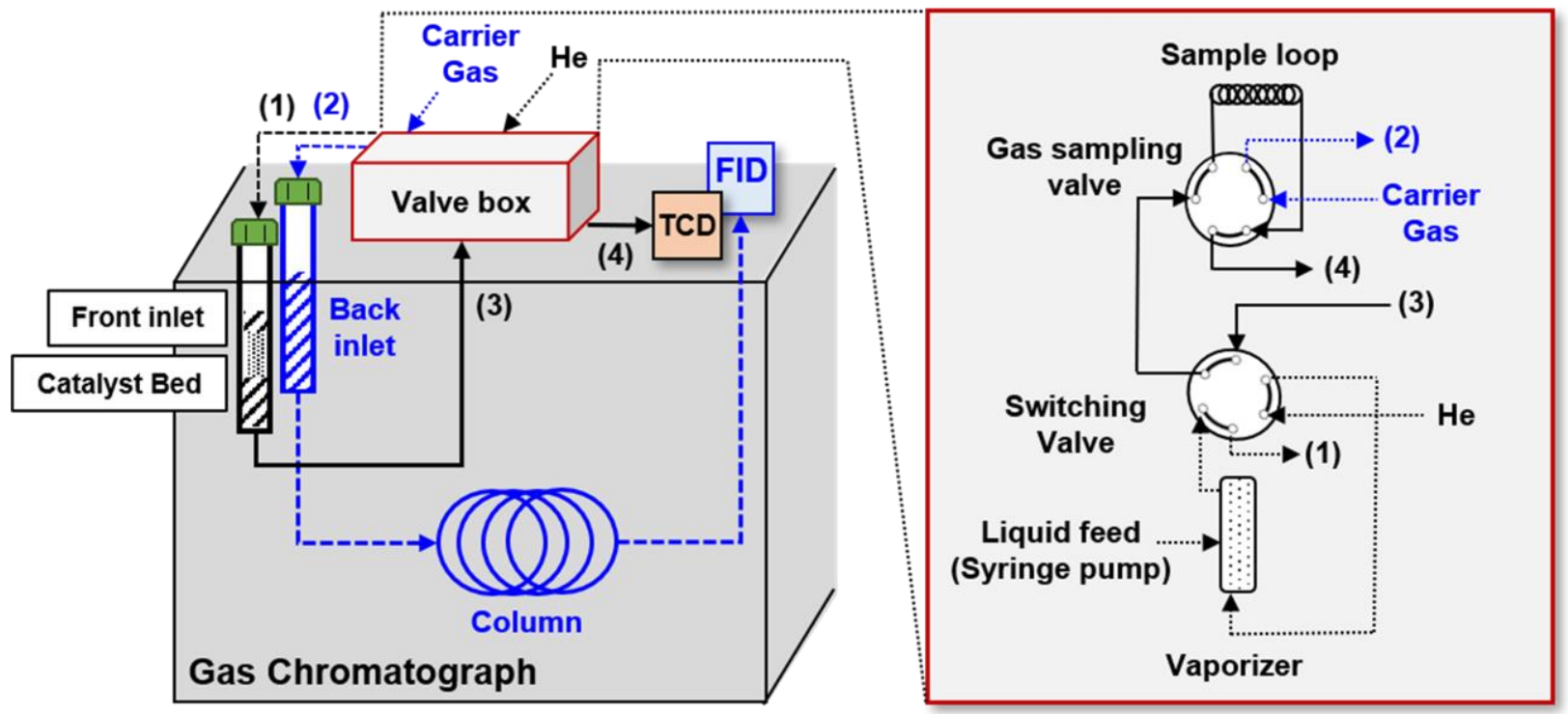

Scheme 1. A schematic for the vapor-phase micro-flow reactor integrated within a gas chromatograph (GC). The instrumentation of the setup consists solely of a typical gas chromatograph, where the front inlet is used as a packed bed reactor. The gas flows are routed to either bypass or contact the catalyst bed by using a combination of two six-port valves housed within a heated valve box unit, and a thermal conductivity detector (TCD) is used for online reactor effluent monitoring. The back inlet is utilized for effluent quantification and consequently rate measurements through periodic gas-sampling followed by separation using a GC column and quantification through a flame ionization detector (FID).

\subsection{Materials and Methods.}

2.1 Methods. This section discusses a detailed design and implementation of the reactor, independent temperature measurements to investigate axial variations under non-reaction conditions, and residence time distribution studies within the micro-flow reactor. The methodology used in the apparent kinetic measurements for all the example reactions are also discussed. Detailed heat and mass-transfer characteristics specific to each of the probe reactions are discussed in the SI (Section S.4).

2.1.1 Design and Implementation. The following modifications were performed on an Agilent 7890 GC unit to convert it to the microflow reactor (Scheme 1). The front inlet of the GC was installed as an auxiliary heater. The flow control to this front inlet (housing the catalyst bed) was achieved using upstream referenced mass flow controllers (MFC, Vici Valco Model 100) in conjunction with an auxiliary electronic pressure controller (EPC, Agilent G3452) (gas delivery details can be found in Section S1, and Figure S4). Given the upstream reference configuration of the MFC, a fixed and repeatable gas flow rate could be delivered for a particular supply pressure set by the EPC. Gas flowrates delivered by the MFC were therefore calibrated as a function of the auxiliary EPC pressure set point, which could be directly controlled through the GC (Figure S4).

A splitless inlet liner (Agilent, 5190-2293) was utilized as the reactor tube (I.D. $=4 \mathrm{~mm}$ ) (Figure S2). The split vent for the front inlet was blocked using a capping nut (Swagelok SS-200-P); doing so forced gas flows exiting the reactor through one single outlet (Figure S1 and S3). The back inlet was used for chromatography, where analyte separation was performed by an HP-PLOTQ column (Agilent, 19091P-QO4), connected to a quantitative carbon detector (QCD, Polyarc $\left.{ }^{\mathrm{TM}}\right)^{34}$ in conjunction with a flame ionization detector (FID).

Two six-port valves (Vici Valco, DC6UWE) (shown in Scheme 1; identified as V-1 and V-2 in Scheme 2) housed within a heated valve box (Agilent G1581A) were used to route gas flows and perform gas-phase injections on the back inlet utilized for chromatography. Valve V-1 was installed as a gas-sampling valve; V-2 was installed as a switching valve (Scheme $\mathbf{2}$ and Figure S1). A pressure gauge upstream of the reactor was used to 


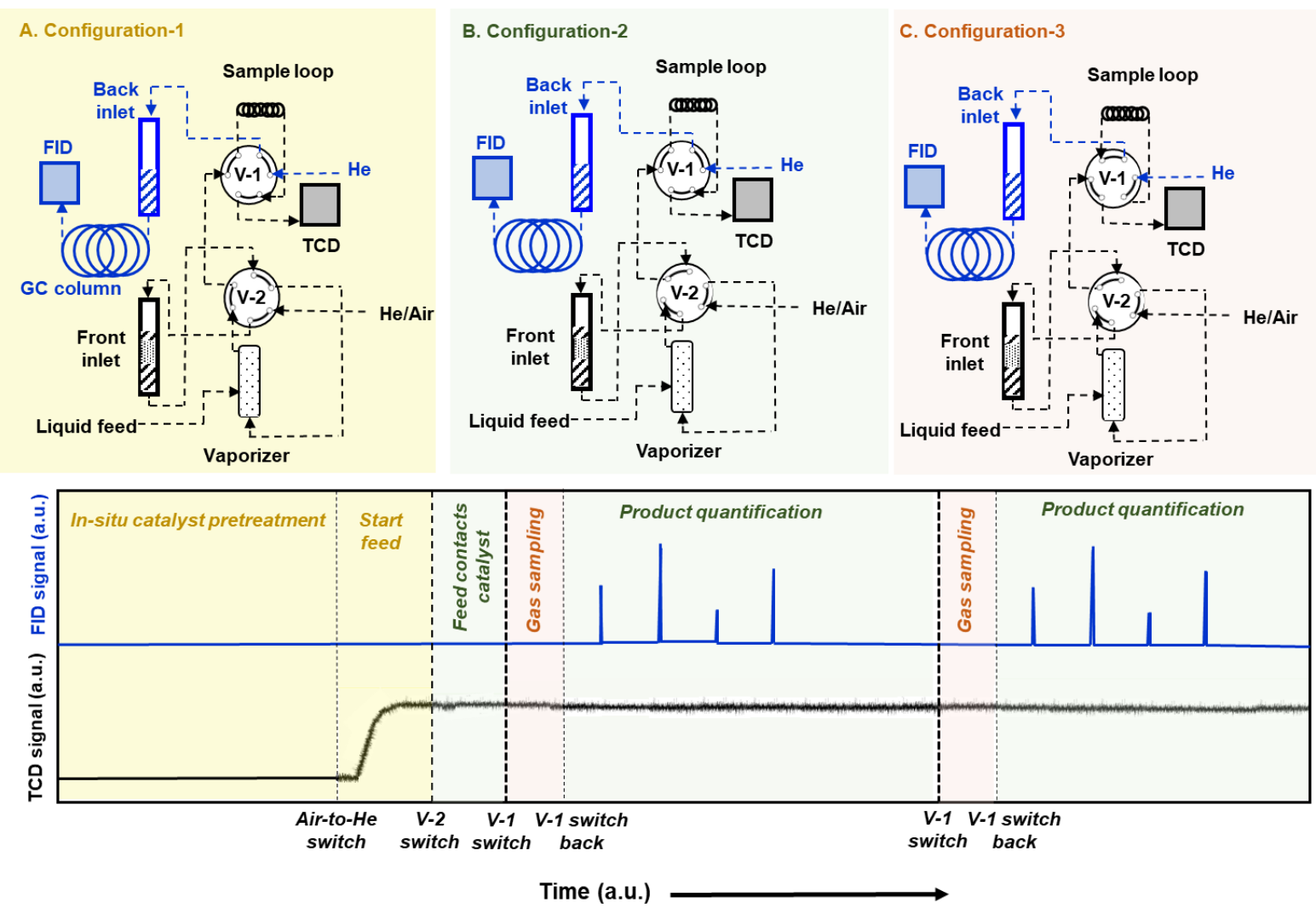

Scheme 2. Methodology for vapor-phase micro-flow reactor integrated within a gas chromatograph (GC). Catalyst samples are loaded into the front inlet liner of a GC and reaction kinetics are studied by operating the setup in three configurations (i) in-situ catalyst pre-treatment mode (Configuration 1; No feed (A)): Catalysts are calcined in-situ in flowing air at $673 \mathrm{~K}$ and then cooled down to reaction temperature and the carrier gas is switched from Air to He; (ii) Reactant dosing in bypass mode (Configuration 1; Feed on (A)): A syringe pump delivers a steady flow of liquid reactant to the vaporizer, bypassing the reactor, and is routed directly to the online detector (TCD) till a stable signal is observed; and (iii) Reactant introduction and product quantification mode (Combination of Configurations 2 and 3 (B \& C)): The vaporized reactant stream is contacted with the catalyst bed in configuration 2, followed by periodic gas sampling (configuration $\mathbf{3}$ ) leading to the separation and quantification of products through QCD/FID.

monitor the pressure drops across the catalyst bed, by comparing to a reference pressure measured in absence of a catalyst bed (indicative of pressure drop due to process tubing and fittings). A vaporization section was housed within the heated valve box, and consisted of a $1 / 4$ " tube (316 SS) filled with deactivated quartz chips $\left(\mathrm{SiO}_{2}, 4-20\right.$ mesh, Sigma Aldrich) to facilitate static mixing and effective vaporization of the injected liquid (Figure S1). A thermal conductivity detector (TCD) placed in-line with the flow exiting the reactor stream was used as an online detector (Scheme 2).

Both front and back inlets were insulated with fiberglass insulation sheet (McMaster) to ensure that bed temperatures were close to the inlet wall temperature controlled by the auxiliary heater; this was later confirmed by secondary measurements on an empty bed (discussed in Section 3.1).

Scheme 2 depicts the overall experimental methodology for performing kinetic measurements using the setup in three different configurations which are discussed below. The different valve positions to operate the setup for these specific configurations are also discussed, and listed in Table 1.

(a) Catalyst pre-treatment mode (Configuration 1; No reactant feed): Catalyst powders were pressed and sieved into aggregates of 500-1000 $\mu \mathrm{m}$ and placed between deactivated quartz wool (Restek 24324) to keep the catalyst bed in place. Typically, $\sim 25-30 \mathrm{mg}$ catalyst was used for the kinetic measurements. Catalyst masses were 
Table 1. Possible valve positions and the corresponding configurations as highlighted in Scheme 2.

\begin{tabular}{|c|c|c|c|c|}
\hline \multicolumn{2}{|c|}{ Valve position } & \multirow[b]{2}{*}{ Gas } & \multirow[b]{2}{*}{ Function } & \multirow[b]{2}{*}{ Configuration } \\
\hline V1 & V2 & & & \\
\hline Off & Off & Air & Calcination & 1 \\
\hline Off & Off & $\mathrm{He}$ & Bypass & 1 \\
\hline Off & On & $\mathrm{He}$ & Reaction & 2 \\
\hline On & On & $\mathrm{He}$ & $\begin{array}{c}\text { Reaction - } \\
\text { Sample } \\
\text { injection }\end{array}$ & 3 \\
\hline
\end{tabular}

restricted to keep the bed located in the normalized height $\mathrm{h} / \mathrm{H}$ ( $\mathrm{h}$ is the height for packing the bed; $\mathrm{H}$ is total height of the liner) range of $0.15-0.35$, where differences between actual and GC indicated temperature were found to be negligible; this is discussed in more detail in Section 3.1. Following the placement of the reactor in the front inlet, catalysts were pre-treated in air $(99.997 \%$, Minneapolis Oxygen) at $673 \mathrm{~K}$ for five hours at a ramp rate of $3 \mathrm{~K} \mathrm{~min}^{-1}$ (Scheme 2a). After pretreatment, the catalyst was cooled down to reaction temperature, and the gas supply was switched to $\mathrm{He}$ (99.995\%, Matheson). The catalyst was kept at reaction temperature and purged with $\mathrm{He}$ for at least 30 minutes prior to reactant introduction.

(b) Reactor bypass mode (Configuration 1; reactant feed on): In the same configuration, liquid reactants were then pumped into the vaporization section through a $1 / 16$ " PEEK capillary line (0.01" ID) using a syringe pump (74905-04, Cole Parmer) and swept by the He stream exiting the reactor. In this way, the reactant stream bypassed the reactor by placing the vaporization section downstream of the reactor (configuration 1, Scheme 2a). The valve box temperature was maintained at $473 \mathrm{~K}$ to ensure that all species were retained in the vapor phase. The vaporized reactant stream was swept through the vaporization section to V-2 (Scheme 2b) and routed to the TCD after filling the sampling loop located on V-1. Prior to introducing the reactant stream to the reactor, a steady reactant stream was ensured by observing a stable TCD signal for at least 15 minutes. Periodic gas sampling injections in this bypass configuration allowed for the total carbon quantification to gauge carbon balances during reaction.

(c) Reactant introduction and product quantification mode (Combination of Configurations 2 and 3): Once a stable vapor stream of reactant was established, V-2 was switched to direct the flow of He through the vaporizer section first (i.e., after the V-2 switch, the vaporizer becomes upstream of the reactor). The vaporized stream of reactants thus passed through the reactor and contacted the catalyst bed (configuration 2, Scheme 2b). Effluent from the reactor continued to fill the sample loop attached to $\mathrm{V}-1$ and subsequently passed through the online TCD. Quantitative analysis of the reactor effluent was achieved by periodically switching the gassampling valve (V-1) at the start of every chromatography analysis (configuration-3, Scheme 2c). This effectively allowed for the quantitative transfer of information from the reactive front inlet portion of the $\mathrm{GC}$, to the analytical chromatography back inlet portion. Helium carrier gas carried the contents of the sampling loop through the GC column to the QCD/FID for separation and quantification, and V1 switched back after the sample injection was complete $(0.5 \mathrm{~min})$.

Configured this way, all kinetic measurements were performed in downflow mode. Notably, the setup could be operated in either of the three configurations without any manual intervention. This ease of operation was due to the integration of all control elements within the GC circuitry and software without the use of any programmable logic controllers (PLCs) and/or customized LabView programs. These benefits are specifically listed below:

(i) Reaction temperatures could be varied by adjusting the front inlet temperature (installed as an auxiliary heater) in the corresponding method files (ChemStation ver 8.2.1).

(ii) $\mathrm{V}-2$ switching from bypass-to-reactor mode, as well as V-1 injection to the back inlet for product separation/quantification were also controlled directly through ChemStation method files.

(iii) Gas flows were set by calibrating the MFCs on auxiliary EPC (set in method files) with the final flowrates at the TCD outlet.

We note that the methodology described here is for the specific application reported, and can be easily tuned to other applications. For example, the choice of online detector (TCD in this work) is up to the user, and even the absence of an online detector and PolyARC $^{\mathrm{TM}}$ (a quantitative carbon detector) does 
not compromise the performance of the setup. Secondary detectors such as a mass spectrometer can be added to augment the quantitative information with real-time product identification. Similarly, for the reactant delivery, if reactants need to be introduced only for a short period of time (< 5-10 mins), an automated liquid sampler (ALS) can be used for a continuous pumping of reactants and can essentially act as a syringe pump. Indeed, if preliminary product distributions on a variety of different materials is the only intended application, a much simpler version of this setup with pulsed dosing of reactants followed immediately by separation and detection of products can be utilized, as highlighted by our earlier works. ${ }^{26,27}$ While we use syringe pumps for liquid delivery, gaseous reactants can also be dosed using a separate gas line/s equipped with MFC/s. Total reaction pressures in excess of atmospheric levels can be achieved by using back-pressure regulator, and we have been to achieve pressures as high as $~ 100 \mathrm{psi}$ with this simple modification, possibly allowing for moderately high-pressure chemistries to be probed (e.g. hydrodeoxygenation). In short, there are many conceivable modifications using hardware that can be readily integrated with the GC depending on the specific application, rendering this approach versatile.

2.1.2 Temperature variations under nonreacting conditions. Temperature measurements were obtained on the front liner packed with deactivated quartz wool by inserting a 1/16" thermocouple (Omega). This 'empty reactor' was maintained at a particular set temperature as indicated by the GC for at least 20 minutes prior to every measurement, and the measurements were repeated in at least 10 different axial positions along the length of the liner.

\subsubsection{Hydrodynamic behavior under non-} reacting conditions. Residence time distributions (RTD) were measured to establish the range of flowrates under which flow in the setup was sufficiently plug-flow. All residence time studies were conducted by connecting the reactor effluent directly to the inlet of the TCD, so as to minimize the volume between the point of injection and detection. The reactor inlet was packed with deactivated quartz wool and maintained at $523 \mathrm{~K}$; pulses of liquid ethanol were used as tracer. Liquid injections of ethanol pulses were manually performed with a $0.5 \mu \mathrm{L}$ syringe (Agilent). Tracer identity is unlikely to change the hydrodynamic behavior under non-reacting conditions, ${ }^{35}$ and the results obtained with ethanol are assumed to be broadly applicable. Typical injection volumes were $0.4 \mu \mathrm{L}$, and the experiments were conducted at low carrier flowrates $(13-40 \mathrm{sccm})$ to capture the transition from axially dispersed to a non-dispersed regime.

2.1.4 Catalytic evaluation. Comparison of the turnover rates of reactions involves the calculation of site time yields (STYs) (Eq. 1). For Brønsted acid catalysed chemistries, this calculation requires normalizing mass-based rates of reaction by the total Brønsted acid site density (BAS). The Brønsted acid site density (BAS, $\mathrm{N}_{\mathrm{H}}^{+}$) of the catalyst (HZSM-5) was measured by the quantification of butenes resulting from the Hofmann elimination of tert-butylamine using reactive gas chromatography (RGC) methodology described elsewhere, ${ }^{36}$ and the results are discussed in the supporting information (Section S.2). These values $\left(\mathrm{N}_{\mathrm{H}}^{+}\right)$were used in the calculation of sitetime yields (Eq. 1) in all probe reactions.

$$
S T Y_{i}=\frac{F_{i}}{m_{c a t .} N_{H^{+}}}=\frac{\left[\text { mol product }_{i} / h\right]}{[\mathrm{g}]\left[\mathrm{mol} \mathrm{H}^{+} / \mathrm{g}\right]}
$$

All kinetic experiments were performed under near-differential conversions $(<\sim 15 \%)$ for a given feed by adjusting the weight-hourly space velocities (WHSVs). The reactions were investigated at $\sim 25$ torr partial pressure of reactant. Alcohol dehydration reactions were carried out in the temperature range $388 \mathrm{~K}-483 \mathrm{~K}$, whereas 2methyltetrahydrofuran (2-MTHF) dehydradecyclization was conducted in the temperature range 463-543 K. Due to an abundant literature on alcohol dehydration on solid acids, kinetic data obtained from the micro-flow reactor were compared with previously reported values. For the case of 2-MTHF dehydra-decyclization, however, the kinetic parameters obtained on the micro-flow reactor were directly compared with measurements performed on a traditional packed bed reactor as described in our earlier work. ${ }^{37}$ Minimal catalyst deactivation was observed after initial transients for alcohol dehydration kinetics under the investigated conditions, and the rates reported here are steadystate values. Replicate experiments were carried out using randomized reactor temperature sequencing 


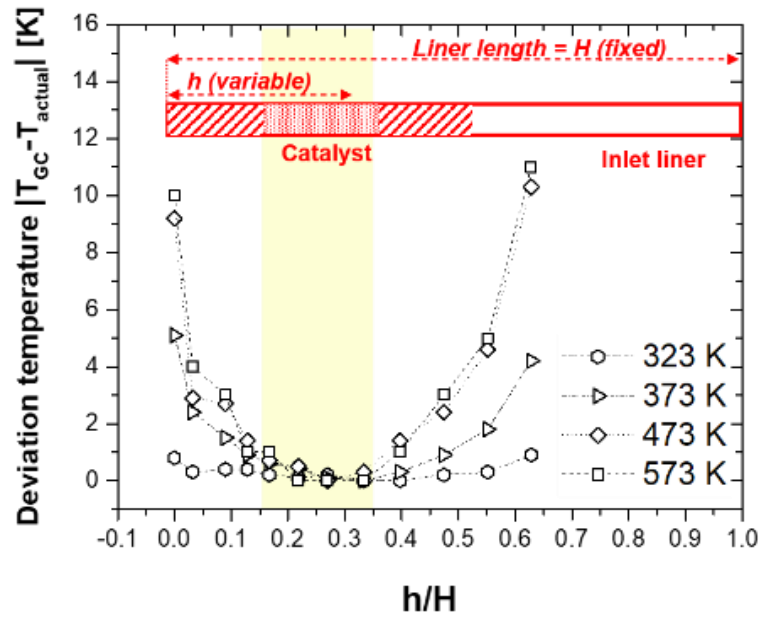

Figure 1. The deviation in set and actual temperature as a function of the non-dimensionalized height of the splitless inlet liner used to hold the catalyst bed.

to minimize any systematic errors. Significant catalyst deactivation was observed during 2-MTHF dehydra-decyclization, and the methods to correct for deactivation to report initial rates are described in detail in Section S.3.

All carbon balances closed to within $\pm 10 \%$. Pressure drops across the reactor were maintained below 13-16\% of total pressure for all kinetic experiments. Error bars represent the 95\% confidence interval on at least three independent measurements, unless otherwise stated.

2.1.5 Data automation. The data analysis tool reported in this work is capable of processing data files (.ch files) from the Agilent Gas Chromatograph with ChemStation as well as OpenLab CDS ChemStation software. We note that these are the most commonly used GC and chromatography analysis software packages. The source code for this tool is available on GitHub, ${ }^{38}$ and the details of raw data parsing, peak detection, and peak area calculation and other analysis pipeline steps, along with illustration of each step, can be found in the Supplementary Information (SI, Section S.6).

2.2 Materials. Ethanol (200 Proof, $\geq 99.5 \%$, Sigma Aldrich), 2-propanol ( $\geq 99.5 \%$, Sigma Aldrich), 1-butanol ( $\geq 99.5 \%$, Sigma Aldrich), and 2-methyltetrahydrofuran (2-MTHF, $\geq 98 \%$, stabilized with BHT, TCI Chemicals) were used without further purification. Ammonium form of a high silica ZSM-5 (Si/Al=140) (Zeolyst CBV28014) was calcined ex-situ under airflow $(\sim 40 \mathrm{sccm})$ (zero grade, Minneapolis Oxygen) at
$823 \mathrm{~K}$ for 10 hours at a ramp rate of $3 \mathrm{~K} \mathrm{~min}^{-1}$ (Lindberg Blue $\mathrm{M}$ tubular furnace). An exhaustive list of all major instrumentation parts required to fabricate the setup is included in the SI (Section S.1, Table S.1).

\subsection{Results and Discussion.}

3.1 Reactor temperature distribution unreactive conditions. Laboratory-scale fixed-bed reactors conventionally involve heat transfer across a temperature-controlled wall, and gradients between the bulk phase in the reactor and the wall might lead to non-isothermal conditions inside the reactor. Similarly, a GC inlet is heated by a cartridge heater, and the controlled temperature is that of the inner inlet wall. To gauge the differences between the differences between the reactor set temperature and the actual temperature of the catalyst bed, independent measurements at different temperature set points as a function of axial positions inside the liner were conducted, and the results are shown in Figure 1. Notably, the axial region in the range $0.15<\mathrm{h} / \mathrm{H}<0.35$ showed $<1 \%$ difference between measured and set temperature under non-reacting conditions. Furthermore, the deviation in temperature from the setpoint outside this region was significantly higher $(>10 \%)$ at higher temperatures (> $473 \mathrm{~K})$. These results highlight that the axial location of the catalyst bed is sensitively linked to the temperature control attainable on this setup.

It is important to note that these measurements only probe the temperature distribution of the reactor under non-reacting conditions. Temperature gradients across the bed as well as within catalyst particles may also develop under reacting conditions, depending on reaction thermodynamics. As expected, highly endo/exothermic reactions are more likely to result in axial temperature gradients. ${ }^{39}$ We have evaluated the temperature distribution of the catalyst bed under the reaction conditions for the probe reactions, and the results are discussed in Section 3.3.

3.2 Residence time distributions. Axial mass dispersion in packed bed reactors can limit the accurate determination of kinetic parameters. This manifests in the form of channeling and dead volumes, leading to back-mixing and non-plug flow hydrodynamics. ${ }^{40}$ The extent of dispersion is described by, and inversely proportional to, the 

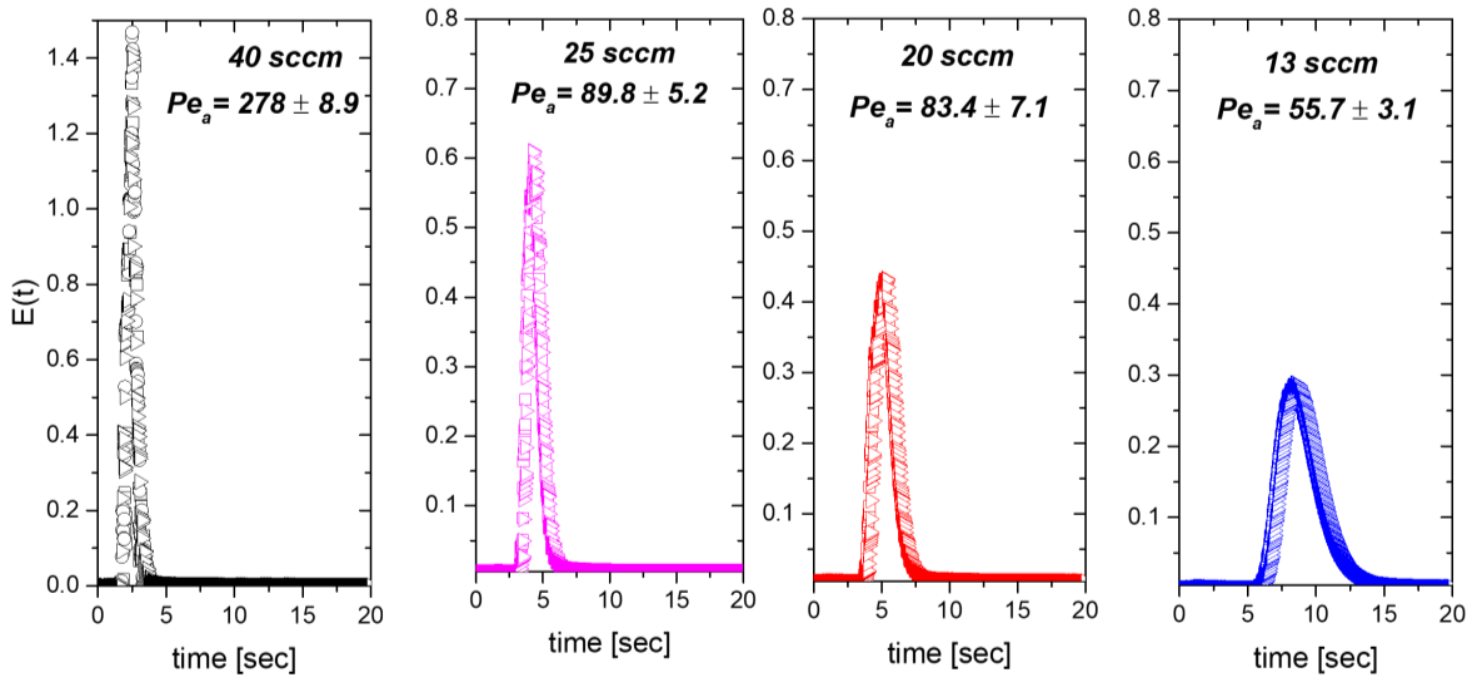

Figure 2. The residence time distribution obtained from the inlet liner packed with deactivated quartz wool at four different carrier gas flowrates. The experiments were performed with ethanol pulses and the inlet was maintained at $523 \mathrm{~K}$. Different symbols ( $\square, \circ$, and $\triangleright$ ) represent replicate runs under identical conditions. Corresponding Peclet numbers given by Eq. 6 are also indicated on the insets. Errors indicate the 95\% CI on the Peclet numbers resulting from independent experimental runs.

Peclet number (Eq. 5), and packed beds can only be assumed to be free from axial dispersion effects for $\mathrm{Pe}>100{ }^{40}$ The extent of axial dispersion is inversely proportional to the bed length, and RTD experiments were therefore performed on the reactor packed with quartz wool alone mimicking the limit $L \rightarrow 0 .^{34}$

Figure 2 depicts the experimental residence time distributions (RTDs) obtained at different gas flowrates. The obtained experimental data was fit to the RTD distribution of an axially dispersed plug flow reactor model with open boundary conditions (Eq. 2). Using the average residence times (Eq. 3), experimental variances (Eq. 4) were then compared with the corresponding values derived from the model (Eq. 5) to calculate Peclet numbers (Eq. 6).

$$
\begin{aligned}
& E(t)=\sqrt{\frac{u^{3}}{4 \pi D L}} \exp \left[-\frac{(L-u t)^{2}}{4 D L / u}\right] \\
& \bar{t}=\int_{0}^{\infty} t E(t) d t \\
& \sigma_{\text {exp }}^{2}=\int_{0}^{\infty}(t-\bar{t})^{2} E(t) \cdot d t \\
& \sigma_{\text {model }}^{2}=\frac{2 D L}{u^{3}}
\end{aligned}
$$

$$
P e_{a}=\frac{u L}{D}
$$

Gas flowrates $\leq 25 \mathrm{sccm}$ led to Peclet numbers in the range $\sim 50-90$, indicating non-negligible degree of back-mixing under these flowrates. However, the RTD curve at a slightly higher flowrate $(40 \mathrm{sccm})$ exhibited significantly lower variance, leading to $\mathrm{Pe} \sim 280$. It is therefore possible to operate the setup with plug flow hydrodynamics at moderately low flowrates (> 40 scem).

3.3 Reaction kinetics measurements. Any new reactor needs to be characterized and benchmarked against traditional designs; this can be accomplished by measuring and comparing reaction kinetics for relatively simple and well understood probe chemistries. To this extent, we utilized C2-C4 alcohol dehydration, and the dehydra-decyclization of a five-membered saturated ether 2-MTHF. Alcohol dehydration on Brønsted acid sites proceeds by two mechanisms: a unimolecular pathway to the corresponding olefin, and a bimolecular pathway to a di-alkyl ether (Scheme 3). ${ }^{41-44} \quad$ Alternatively, 2methyltetrahydrofuran (2-MTHF) can either undergo dehydra-decyclization to linear pentadienes, namely 1,3-pentadiene and 1,4pentadiene, or fragment to butenes and formaldehyde by a competing retro-Prins 


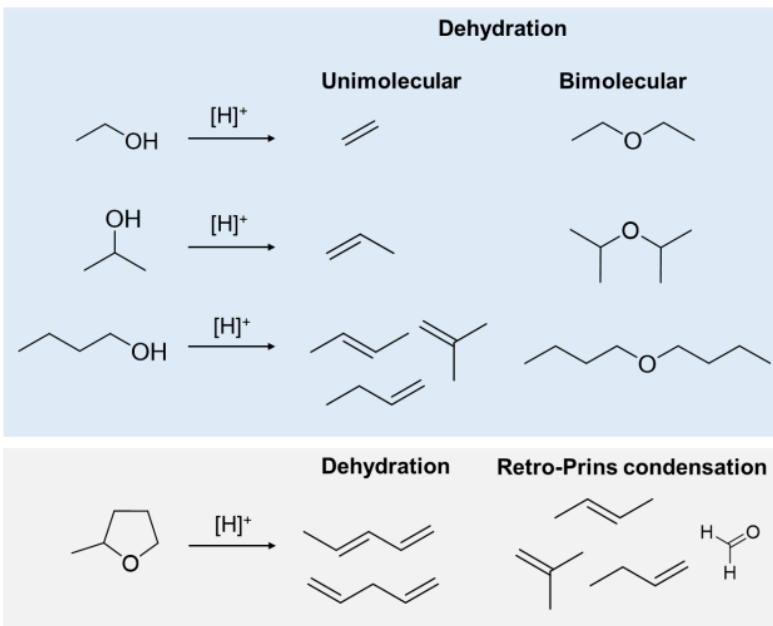

Scheme 3. Known reaction pathways for the Brønsted acid catalysed dehydration of ethanol, 2-propanol, 1butanol, and dehydra-decyclization of 2methyltetrahydrofuran (2-MTHF).

condensation pathway (Scheme 3). Other typical side products are propene and large aromatics (C6+ fraction). ${ }^{45}$

In the case of alcohol dehydration, the kinetic preference to unimolecular/biomolecular dehydration pathways was found to be temperature dependent. Increasing temperature consistently led to unimolecular dehydration being favored over the

A.

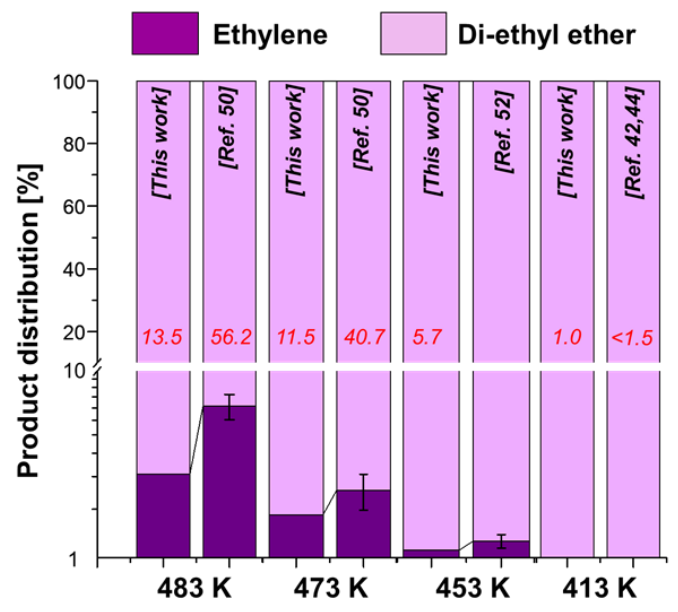

bimolecular pathway for all three alcohols. Taking ethanol as a representative case, this observation is highlighted in Figure 3A. While di-ethyl ether was the only observed dehydration product at low temperatures $(\leq 413 \mathrm{~K})$, ethylene selectivities exhibited a monotonic rise with increase in temperature for the range investigated (388 K-483 $\mathrm{K})$. Corresponding product distributions from previous reports are also shown for the same chemistry in Figure 3A , and direct comparisons reveal good agreement between the product distributions obtained on the micro-flow reactor setup and previously reported values. It has been widely reported that the 10 -membered ring (MR) channels in the MFI framework (for ZSM-5) enthalpically stabilize the bulkier bimolecular pathway transition state (TS) for light alcohol (C1C4) dehydration at low temperatures due to tighter pore confinement. ${ }^{41,42,44,46}$ Increasing temperature increases the contribution of entropy, thereby favoring looser transition state fits, and leading to higher unimolecular product selectivities at higher temperatures. Consistent with this discussion, the same behavior was indeed observed for the other two alcohols (namely, 2-propanol and 1-butanol)

Figure 3. (A) The product distribution obtained from ethanol dehydration at different reaction temperatures compared with previously reported values on HZSM-5. The conversions are indicated on the chart in red; the values for Ref.52 were not explicitly reported but differential (low). WHSV for the data from the micro-flow reactor is $7.35 \mathrm{~g}$ EtOH/g cat./h; (B) The product distribution obtained from 2-MTHF dehydra-decyclization at different reaction temperatures compared with measurements carried out on HZSM-5 (Zeolyst CBV8014, Si/Al=40) under similar experimental conditions on a traditional packed bed reactor (PBR) as described in Ref. 37. The conversions are indicated on the chart in red. WHSV for the data from the micro-flow reactor is $5.5 \mathrm{~g} \mathrm{2-MTHF/g}$ cat./h, while WHSV for the data from the PBR is in the range 3.2-7.5 g 2-MTHF/g cat./h). Carbon balances for both sets of data are within $\pm 7 \%$. 
Table 2. Apparent activation energies for the major dehydration product of ethanol, 2-propanol, 1-butanol, and 2MTHF under reaction conditions measured on HZSM-5 (Si/Al 140) compared with previously reported values.

\begin{tabular}{|c|c|c|c|}
\hline \multirow[b]{2}{*}{ Reactant } & \multirow[b]{2}{*}{ Product(s) } & \multicolumn{2}{|c|}{ Apparent activation barrier [kcal/mol] } \\
\hline & & This work & Literature \\
\hline Ethanol & di-ethyl ether & $19.7 \pm 1.5$ & $21.9^{50}, 23.2^{44}, 24.5^{42}$ \\
\hline 2-propanol & propene & $34.5 \pm 1.9$ & $34.9 \pm 1.6^{36}$ \\
\hline 1-butanol & di-butyl ether & $17.6 \pm 0.7$ & $18 \pm 2^{51}, 21.0 \pm 0.8^{37}$ \\
\hline 2-methyltetrahydrofuran & $\begin{array}{l}(1,3+1,4)- \\
\text { Pentadienes }\end{array}$ & $17.4 \pm 1.9$ & $21.5 \pm 1.5^{*}, 17.7 \pm 1.7^{47 * *}$ \\
\hline
\end{tabular}

* These measurements were performed on a traditional packed bed reactor as described in Li et al. ${ }^{37}$

**These measurements were performed on amorphous silica-alumina (13.7 wt \% alumina content)

employed in this study, and the results can be found in Table S3 and Section S4.

For 2-MTHF dehydra-decyclization, linear pentadienes were found to be the dominant product with selectivities of $\sim 70-75 \%$ under low conversions $(2.9-8.0 \%)$ in the temperature range investigated (483-523 K) (Figure 3B). The obtained product distributions were again found to be in reasonable agreement with the values obtained from the traditional packed bed reactor setup, as well as previously reported values under similar reaction conditions. ${ }^{47}$

The Arrhenius dependence of protonnormalized rates of the most dominant reaction products for all the test reactions are plotted in Figure 4. The corresponding values of STYs previously reported under similar experimental conditions are also plotted to directly compare them against the values obtained from the micro-flow reactor setup. The STYs for all the probe reactions obtained from our setup are typically within a factor of $\sim 2 \mathrm{x}$ of the corresponding values reported in literature. The calculation of STYs inherently has considerable errors associated with it, in part due to the different measurement methods for estimating the Brønsted acid site count. Furthermore, the absolute value of STYs depend on the experimental variables like the space velocities and consequently
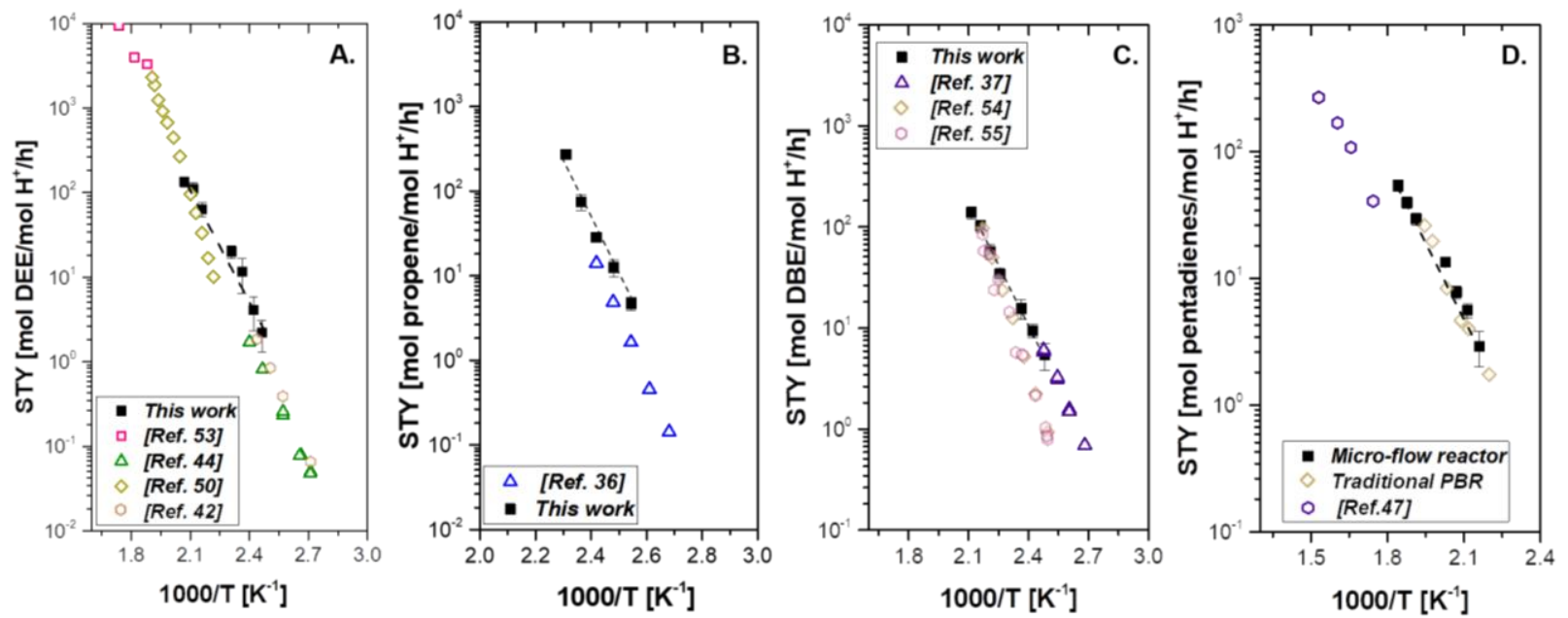

Figure 4. Site-time yields of major products plotted as a function of inverse temperature for (left to right) (A) ethanol, (B) 2-propanol, (C) 1-butanol, and (D) 2-MTHF (Reaction conditions: $P_{\text {reactant }} \sim 25$ torr, He flowrate $=60$ sccm, WHSVs in the range 5.0-7.6 g reactant/g cat./ h; all conversions kept below 15\%). Error bars in A-C represent the 95\% CI on multiple injections from the same experimental run to measure steady state rates, while the error bars in D represent $95 \%$ CI on replicate independent measurements on fresh/recalcined catalyst beds. The references indicated on the insets are also listed in the last column of Table 2. 


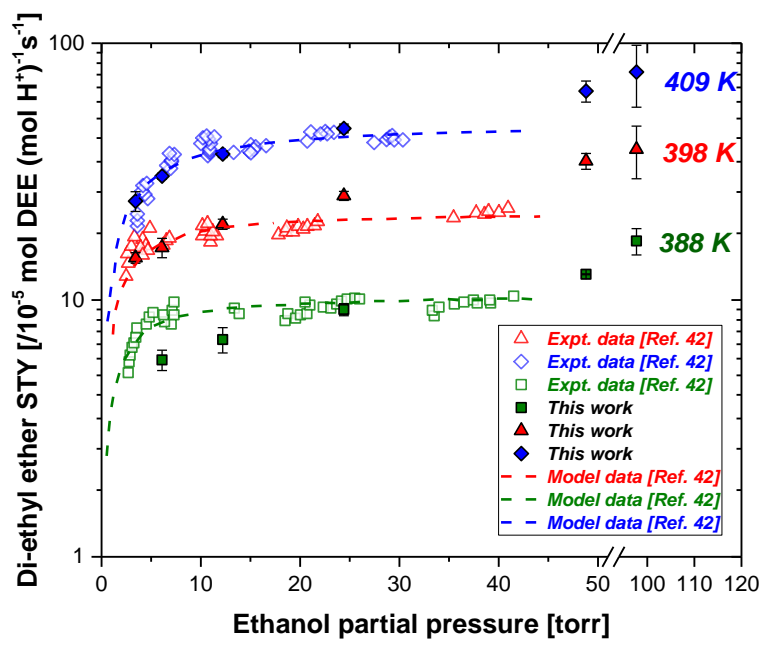

Figure 5. Di-ethyl ether site-time yields measured as a function of ethanol partial pressure on HZSM-5 (Si/Al 140) at $388 \mathrm{~K}, 398 \mathrm{~K}$, and $409 \mathrm{~K}$, respectively, on the micro-flow reactor setup. The error bars represent a 95\% CI in the steady state rate measurements on one catalyst bed (Reaction conditions: WHSVs in the range 0.81-28.6 g EtOH $/ \mathrm{g}$ cat $/ \mathrm{h}$, carrier gas flowrate $=60$ sccm, all conversions were kept below $1.2 \%$ ). The reported data is compared with experimental and modeled data from Chiang et al. ${ }^{42}$ where the rates were reported at conversions $<1.5 \%$ on HZSM-5 (Si/Al 42.5).

the conversions at which the product formation rates are calculated, and hence it is safe to conclude that the micro-flow reactor reported here is capable of accurate STY measurements provided the conversions are near-differential $(<15 \%)$. The apparent activation barriers extracted from the Arrhenius plots measured in this work are listed in Table 2 along with previously reported values. Considering the apparent nature of our measurements (which are likely a convolution of intrinsic kinetics and thermodynamics), the agreement with previously reported values is also reasonable, especially given that some of these referenced values are intrinsic zero-order rateconstants, and are expected to be higher than apparent (nearly first-order) rate constants due to adsorption enthalpy contributions incorporated in first-order rate constants.

Another application of a laboratory-scale PBR is the estimation of rate orders by varying reactant partial pressures. We highlight the applicability of the micro-flow reactor setup by considering ethanol dehydration at low temperatures (388-409 K); under these conditions, di-ethyl ether (DEE) is the only reaction product. As expected, DEE production rates were sensitive to the ethanol partial pressures in the low partial pressure regime ( $<10$ torr) (Figure 5). At high ethanol partial pressures ( $>30$ torr), DEE production rates remained nearly invariant with increasing ethanol partial pressures, indicating a nearly zero-order kinetic regime conferred by a surface saturated with ethanol. The absolute DEE STY values as well as ethanol partial pressure dependence showed reasonably good agreement with values previously reported under similar reaction conditions on ZSM5 by Bhan and co-workers. ${ }^{42}$ Along with all the applications highlighted so far, long-term stability analysis of a catalyst can also be performed on this setup, provided that the reaction conditions ensure complete bed utilization. ${ }^{48}$ An example case for a test chemistry suffering from deactivation (2MTHF dehydra-decyclization) carried out on HZSM-5 is included in the SI (Section S5 and Figure S6) to illustrate the efficacy of this setup to perform automated long-term stability investigations.

As previously noted, calculations to probe the temperature distribution of the reactor under reaction conditions were carried out by: (i) the measurement of axial bed temperature gradients, combined with (ii) calculation of inter-, and intraparticle temperature gradients (Section S.4 in the SI). For the reported kinetics collected under strictly differential conversions, both external and internal particle gradients were found to be negligible (Section S.4 in the SI). At the highest temperature of kinetic measurements, the axial temperature change across the catalyst bed were estimated to be $\sim 0.6 \mathrm{~K}, \sim-2.4 \mathrm{~K}, \sim 0.7 \mathrm{~K}$, and $\sim 0.4$ $\mathrm{K}$ for dehydration of ethanol, 2-propanol, 1butanol, and 2-MTHF, respectively (see Section S.4 in the SI). With the exception of $2.4 \mathrm{~K}$, these temperature drops are all within the resolution limits of a thermocouple and indicate the nearly isothermal operation of the reactor, at least under these low conversion conditions.

These results, taken together, highlight that the product distributions, absolute values of STYs along with their partial pressure dependencies, and the apparent activation barriers measured using this setup are under nearly isothermal reaction conditions, and hence in good agreement with previous studies reported on more traditional packed bed reactors. Comparable reactor 


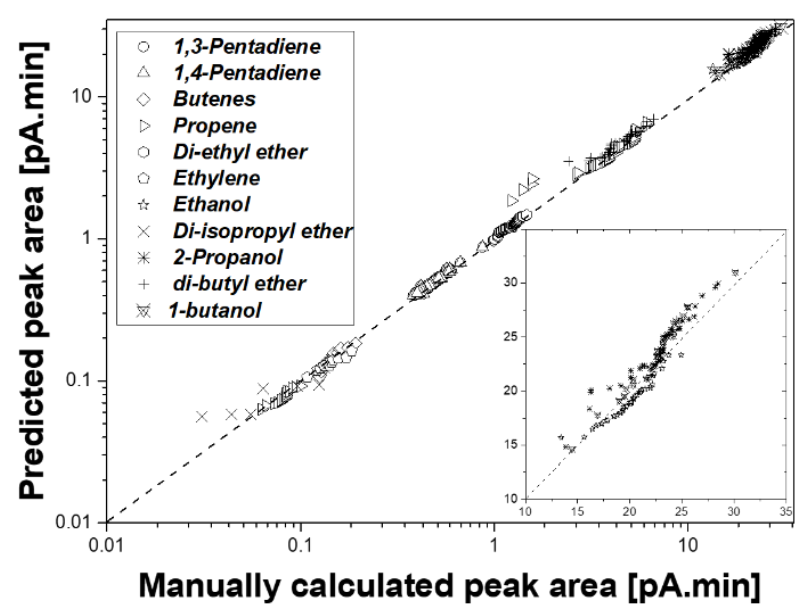

Figure 6. Parity plot comparing the peak areas of different compounds calculated by the Chromatography analysis tool viz-a-viz manually calculated values. Inset shows a magnified view of the data corresponding to high area values (10-35 a.u.) on a linear scale.

performance underpins the utility of this automated setup to obtain kinetic parameters.

3.4 Data automation. The developed chromatogram analysis tool allowed parsing the raw (.ch) files generated from the FID through the analysis pipeline as described in Section S.6 to detect chromatogram peaks and calculate peak areas. The peak areas calculated with this tool were then compared with manually calculated peak areas to investigate its reliability in data analysis. Data of the analytes from the four probe reactions resulted in a total of $>200$ data points, spanning more than two orders of magnitude, and the results are presented in the parity plot shown in Figure 6. There was generally good agreement between the manually calculated areas and the corresponding values predicted by the tool irrespective of compound identity, although the values for manual integration were found to be scaled down by a fixed factor. The presented results indicate that the chromatography analysis tool developed can be effectively utilized to analyse raw data files generated by this automated reactor system.

3.5 Benefits and limitations of the reported micro-flow reactor setup. As highlighted through the earlier discussions, the micro-flow reactor affords automated operation without requiring constant human intervention. In addition, the fabrication of the setup is fairly straightforward and requires minimal added instrumentation over and above typical GC operation. Many catalysis laboratories across the world are indeed equipped with fully automated state-of-the-art setups operating in a 24/7 mode, but the key benefit of using the reported approach vs. these high-end commercial reactor systems lies the significant cost savings in implementing this approach. As shown in Figure 7, depending on the desired level of instrumentation (e.g. new/refurbished GC, or the presence/absence of online detectors), the cost benefit of fabricating the micro-flow reactor setup can range anywhere from $\sim 2-10 \mathrm{x}$ over these commercial systems, which amount to cost savings of $\$ 20,000-80,000$. Therefore, this approach can potentially enable significantly more kinetic data per unit cost without compromising its quality. With the ease of operation, relatively simple fabrication, and low cost, we view this approach as a tool for standardized experimental reaction systems which can be readily utilized by materials synthesis researchers.

One can reasonably argue that only one catalyst can be tested at any given time, limiting the reactor throughput, while also requiring manual intervention for catalyst changeover. However, a typical mid-size heterogeneous catalysis laboratory in the US employs anywhere between two-to-10 gas chromatograph units, some of which can be converted to these automated micro-flow reactors given the minimal additional fabrication costs (Figure 7). Therefore, the possible shortcomings arising from the testing capability being limited to one catalyst at any given time can be solved by added investment enabling operation of multiple units in parallel. With the reduced-workforce regulations where staggered work hours in laboratories is going to be the norm for the foreseeable future, these low-cost automated setups offer another key advantage; to the extent that a laboratory has multiple such units, one researcher can operate $\sim 2-5$ systems (potentially being used on different research projects/chemistries/researchers) at a given time depending on familiarity and knowhow, meaning that multiple researchers do not need to be present for 'their' systems to operate in $24 / 7$ mode.

What about the gas cylinder changeovers and setting up feed-stream dosing (which are not controlled by a GC)? With the increasing global shortage of helium, researchers are moving to low cost alternatives like hydrogen for carrier gases for their gas chromatography needs, ${ }^{49}$ which can be 


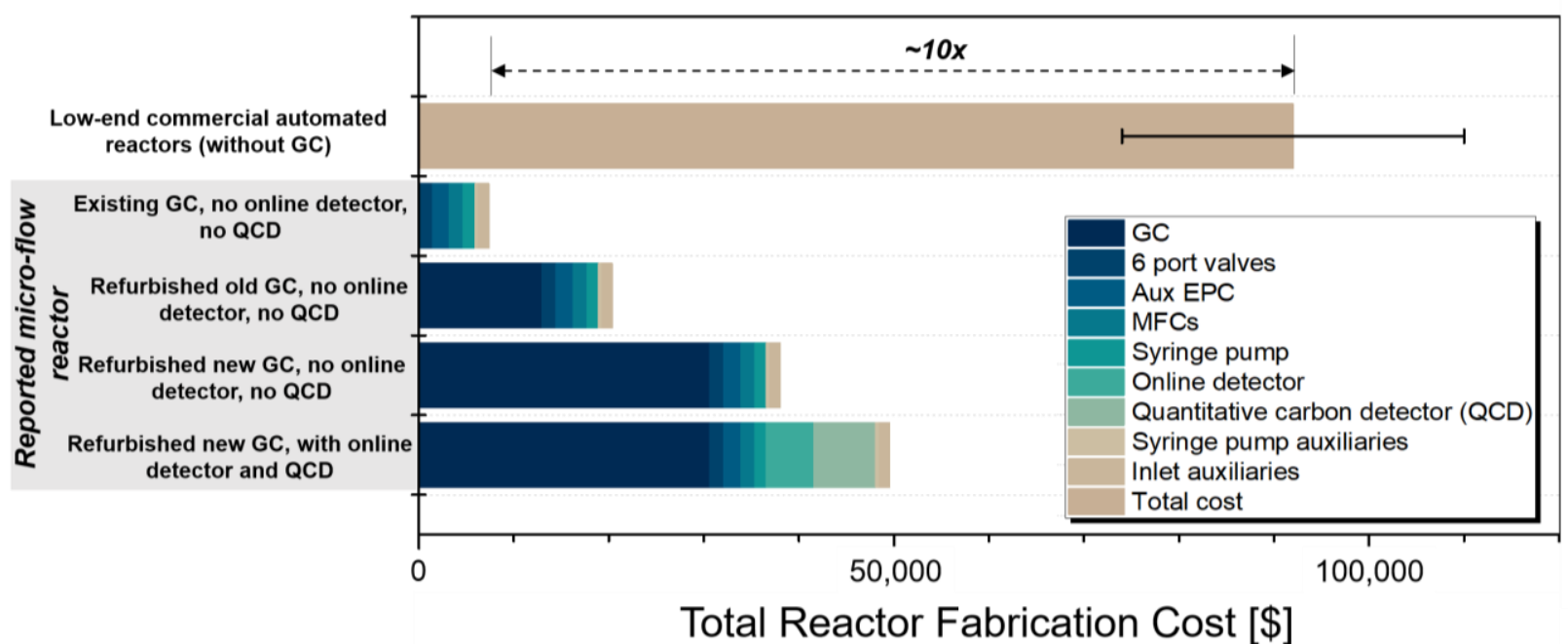

Figure 7. Comparison of the fabrication cost for the reported micro-flow reactor under different considered scenarios (e.g., choice of GC, presence/absence of an online detector and a PolyARC ${ }^{\mathrm{TM}}$ (quantitative carbon detector, QCD) compared with typical prices of commercial low-end automated lab-scale flow reactors.

generated in-house using hydrogen generators. Even in cases where helium gas cylinders are used, it is possible to set up helium cylinder multipacks in conjunction with manifolds for these equipment, enabling continuous operation for weeks without requiring replacement. Setting up liquid feed dosing using one or more syringe/HPLC pumps remains manual but is usually quick; as pointed out before, for applications only requiring an initial estimation of product distributions, a significantly simpler version of this setup as a transient pulsed microreactor from our earlier works ${ }^{26,27}$ can be utilized, which would barely require any manual intervention in setting up feeds as the dosing is through the GC autosampler itself. Similarly, for chemistries utilizing light gases as feeds (viz. methane activation, carbon dioxide valorisation), it is possible to set up individual lines with separate mass flow controllers (MFCs), and such systems can run practically indefinitely for a given catalyst bed.

The ease of fabrication does lead to some limitations of using the proposed system, one of which is the feasible conditions of catalyst temperature control. While a small range of $h / H$ ratios ensures minimal deviation temperature, one should be mindful that temperature deviations can be as high as $>10 \%$ even without considering the reaction thermodynamics, if the catalyst bed packing extends outside the range of $0.15<\mathrm{h} / \mathrm{H}<$ 0.35 . This criterion limits the total mass of catalyst that can be packed in this setup to $\sim 30-45 \mathrm{mg}$, meaning that highly exothermic reactions requiring bed-dilutions may not be suited for this setup. Furthermore, the maximum operating temperature for the setup is limited by the maximum allowable temperature of the GC inlet (673 K, Agilent 7890). Therefore, in-situ catalyst pre-treatments requiring higher temperatures cannot be achieved. Lastly, the unit is not intended to be used for multiphase mixtures; if liquids with low vapor pressures are used, separate heat tracing of individual transfer lines may be required, which compromises the simplicity of fabricating the setup.

4.0 Conclusions. We provide a detailed design and implementation strategy of an autonomous microflow reactor integrated within a typical gaschromatograph unit, enabling kinetic data acquisition without manual intervention for vaporphase chemistries carried out at near ambient pressures. Temperature control inside the reactor depends on the axial position of the catalyst bed, where deviations are negligible in the region $0.15<$ $\mathrm{h} / \mathrm{H}<0.35$. Residence time distribution analysis reveals near plug flow hydrodynamics at relatively small gas flowrates (>40 sccm). A combination of four Brønsted acid catalysed reactions further highlight the absence of heat and mass transport limitations under strictly differential conversions ( $\leq$ $5 \%$ ), and the axial temperature change remains < $2 \%$ for the investigated chemistries. Furthermore, typical kinetic parameters are in reasonable agreement with previous reports. Lastly, a 
chromatogram analysis tool developed in-house integrated with the experimental workflow enables the handling of raw chromatography files for automated evaluation of peak areas. The setup has significant cost savings compared to commercial automated lab-scale reactors, and coupled with its automated implementation affords an opportunity to carry out catalytic evaluation of materials in a manner which is both reliable and consistent with reduced workforce guidelines in place due to COVID-19 pandemic.

5.0 Acknowledgements. We acknowledge financial support of the Catalysis Center for Energy Innovation, a U.S. Department of Energy - Energy Frontier Research Center under Grant DESC0001004.

Keywords. Reactor, Catalyst, Materials, Automation, Robot

Supporting Information. Additional information including fabrication parts and procedures as well as automation instructions can be found in the online electronic supporting information.

\section{References}

(1) Bao, Y.; Leader, G.; Bossion, A. Snapshots of Life Early Career Materials Scientists Managing in the Midst of a Pandemic, Chem. Mater. 2020 Article ASAP (DOI: 10.1021/acs.chemmater.0c01624).

(2) Degnan, T. How will the COVID-19 pandemic affect the catalysis community? Focus Catal. 2020, 2020 (5), 1.

(3) Hartmann, M. Hierarchical zeolites: A proven strategy to combine shape selectivity with efficient mass transport. Angew. Chemie - Int. Ed. 2004, 43 (44), 5880-5882.

(4) Chughtai, A. H.; Ahmad, N.; Younus, H. A.; Laypkov, A.; Verpoort, F. Metal-organic frameworks: Versatile heterogeneous catalysts for efficient catalytic organic transformations. Chem. Soc. Rev. 2015, 44 (19), 6804-6849.

(5) Wang, A.; Li, J.; Zhang, T. Heterogeneous singleatom catalysis. Nat. Rev. Chem. 2018, 2 (6), 65-81.

(6) Furukawa, S.; Komatsu, T. Intermetallic Compounds: Promising Inorganic Materials for WellStructured and Electronically Modified Reaction Environments for Efficient Catalysis. ACS Catal. 2017, 7 (1), 735-765.

(7) Voiry, D.; Shin, H. S.; Loh, K. P.; Chhowalla, M. Low-dimensional catalysts for hydrogen evolution and CO 2 reduction. Nature Chemistry Reviews 2018 (2),
1-17.

(8) Holzwarth, A.; Schmidt, H. W.; Maier, W. F. Detection of catalytic activity in combinatorial libraries of heterogeneous catalysts by IR thermography. Angew. Chemie - Int. Ed. 1998, 37 (19), 2644-2647.

(9) Cong, P.; Doolen, R. D.; Fan, Q.; Giaquinta, D. M.; Guan, S.; Mcfarland, E. W.; Poojary, D. M.; Self, K.; Turner, H. W.; Weinberg, W. H. High-Throughput Synthesis and Screening of Combinatorial Heterogeneous Catalyst Libraries, Angew. Chem. 1999, 4, 483-488.

(10) Hendershot, R. J.; Snively, C. M.; Lauterbach, J. High-throughput heterogeneous catalytic science. Chem. - A Eur. J. 2005, 11 (3), 806-814.

(11) Van Veen, A. C.; Farrusseng, D.; Rebeilleau, M.; Decamp, T.; Holzwarth, A.; Schuurman, Y.; Mirodatos, C. Acceleration in catalyst development by fast transient kinetic investigation. J. Catal. 2003, 216 (1-2), 135-143.

(12) Corma, A.; Serra, J. M. Heterogeneous combinatorial catalysis applied to oil refining, petrochemistry and fine chemistry. Catal. Today $\mathbf{2 0 0 5}$, 107-108, 3-11.

(13) Huybrechts, W.; Mijoin, J. érôme; Jacobs, P. A.; Martens, J. A. Development of a fixed-bed continuous-flow high-throughput reactor for longchain n-alkane hydroconversion. Appl. Catal. A Gen. 2003, 243 (1), 1-13.

(14) Hendershot, R. J.; Lasko, S. S.; Fellmann, M. F.; Oskarsdottir, G.; Nicholas Delgass, W.; Snively, C. M.; Lauterbach, J. A novel reactor system for high throughput catalyst testing under realistic conditions. Appl. Catal. A Gen. 2003, 254 (1), 107-120.

(15) Hahndorf, I.; Buyevskaya, O.; Langpape, M.; Grubert, G.; Kolf, S.; Guillon, E.; Baerns, M. Experimental equipment for high-throughput synthesis and testing of catalytic materials. Chem. Eng. J. 2002, 89 (1-3), 119-125.

(16) Pérez-Ramírez, J.; Berger, R. J.; Mul, G.; Kapteijn, F.; Moulijn, J. A. Six-flow reactor technology a review on fast catalyst screening and kinetic studies. Catal. Today 2000, 60 (1), 93-109.

(17) Moulijn, J. A.; Pérez-Ramírez, J.; Berger, R. J.; Hamminga, G.; Mul, G.; Kapteijn, F. High-throughput experimentation in catalyst testing and in kinetic studies for heterogeneous catalysis. Catal. Today 2003, 81 (3), 457-471.

(18) Parrott, A. J.; Bourne, R. A.; Akien, G. R.; Irvine, D. J.; Poliakoff, M. Self-optimizing continuous reactions in supercritical carbon dioxide. Angew. Chemie - Int. Ed. 2011, 50 (16), 3788-3792.

(19) Waldron, C.; Pankajakshan, A.; Quaglio, M.; Cao, E.; Galvanin, F.; Gavriilidis, A. An autonomous microreactor platform for the rapid identification of kinetic models. React. Chem. Eng. 2019, 4, 1623- 
1636.

(20) Perera, D.; Tucker, J. W.; Brahmbhatt, S.; Helal, C. J.; Chong, A.; Farrell, W.; Richardson, P.; Sach, N. W. A platform for automated nanomole- scale reaction screening and micromole-scale synthesis in flow. Science 2018, 359 (6374), 429-434.

(21) Holmes, N.; Akien, G. R.; Blacker, A. J.; Woodward, R. L.; Meadows, R. E.; Bourne, R. A. Self-optimisation of the final stage in the synthesis of EGFR kinase inhibitor AZD9291 using an automated flow reactor. React. Chem. Eng. 2016, 1 (4), 366-371.

(22) Cherkasov, N.; Bai, Y.; Expósito, A. J.; Rebrov, E. V. OpenFlowChem-a platform for quick, robust and flexible automation and self-optimisation of flow chemistry. React. Chem. Eng. 2018, 3 (5), 769-780.

(23) Reizman, B. J.; Jensen, K. F. An automated continuous-flow platform for the estimation of multistep reaction kinetics. Org. Process Res. Dev. 2012, 16 (11), 1770-1782.

(24) Watanabe, R.; Komatsu, T.; Sakamoto S.; Uranoc, Y.; Noji, H High-throughput single-molecule bioassay using micro-reactor arrays with a concentration gradient of target molecules, Lab Chip 2018,18, 28492853.

(25) https://github.com/richardingham/octopus (accessed Aug 5, 2019).

(26) Abdelrahman, O. A.; Park, D. S.; Vinter, K. P.; Spanjers, C. S.; Ren, L.; Cho, H. J.; Vlachos, D. G.; Fan, W.; Tsapatsis, M.; Dauenhauer, P. J. BiomassDerived Butadiene by Dehydra-Decyclization of Tetrahydrofuran. ACS Sustain. Chem. Eng. 2017, 5 (5), 3732-3736.

(27) Abdelrahman, O. A.; Park, D. S.; Vinter, K. P.; Spanjers, C. S.; Ren, L.; Cho, H. J.; Zhang, K.; Fan, W.; Tsapatsis, M.; Dauenhauer, P. J. Renewable Isoprene by Sequential Hydrogenation of Itaconic Acid and Dehydra-Decyclization of 3-MethylTetrahydrofuran. ACS Catal. 2017, 7 (2), 1428-1431.

(28) Sica, A.M.; Valles, E.M.; Gigola, C.E., Kinetic data from a pulse microcatalytic reactor-hydrogenation of benzene on a nickel catalyst, J.Catal. 1978, 51, 115125.

(29) Deshpande, A.; Krishnaswamy, S.; Ponnani, K. Pulsed Micro-reactor: An alternative to estimating kinetic parameters of non-catalytic gas-solid reactions. Chem. Eng. Res. Des. 2017, 117, 382-393.

(30) Wang, H.; Zhao, P.; Jiang, B.; Liu, W. A pulse reactor-gas chromatographic investigation of the kinetics of catalytic carbon monoxide hydrogenation. J. Mol. Catal. 1992, 71 (3), 357-364.

(31) Bett. J.A.S; Hall, K.W., The microcatalytic technique applied to a zero order reaction: The dehydration of 2-butanol over hydroxyapatite catalysts, J.Catal. 1968, 10, 105-113.

(32) Verma, A., Kaliaguine Estimation of rate coefficients from pulsed microcatalytic reactors:
Oxidation of ethylene over silver catalyst, J.Catal. 1973, 30, 430-437.

(33) Hickman, D. A.; Degenstein, J. C.; Ribeiro, F. H. Fundamental principles of laboratory fixed bed reactor design. Curr. Opin. Chem. Eng. 2016, 13, 1-9.

(34) Maduskar, S.; Teixeira, A. R.; Paulsen, A. D.; Krumm, C.; Mountziaris; Fan, W.; Dauenhauer, P. J.; Quantitative carbon detector (QCD) for calibrationfree, high-resolution characterization of complex mixtures. Lab Chip 2015, 15 (2), 440-447.

(35) Nauman, E. B. Residence time theory. Ind. Eng. Chem. Res. 2008, 47 (10), 3752-3766.

(36) Abdelrahman, O.; Vinter, K. P.; Ren, L.; Xu, D.; Gorte, R. J.; Tsapatsis, M.; Dauenhauer, P. J. Simple Quantification of Zeolite Acid Site Density by Reactive Gas Chromatography. Catal. Sci. Technol. 2017 (7), 3831-3841..

(37) Li, S.; Abdelrahman, O. A.; Kumar, G.; Tsapatsis, M.; Vlachos, D.; Caratzoulas, S.; Dauenhauer, P. J. Dehydra-Decyclization of Tetrahydrofuran on $\mathrm{H}$ ZSM5: Mechanisms, Pathways, and Role of Transition State Entropy. ACS Catal. 2019, 9, 1027910293, DOI: 10.1021/acscatal.9b03129

(38) https://github.com/dauenhauer-umn/micro-flowreactor-paper (accessed March 18, 2020).

(39) F. Froment, G., Bischoff, K.B., Chemical Reaction Engineering, 1974, (ISBN 9780841201491, doi 10.1021/ba-1972-0109).

(40) Levenspiel, O. Chemical Reaction Engineering, 3rd Edition, 1998 (ISBN 978-0-471-25424-9).

(41) Gounder, R.; Iglesia, E. The Roles of Entropy and Enthalpy in Stabilizing Ion-Pairs at Transition States in Zeolite Acid Catalysis. Acc. Chem. Res. 2012, 45 (2), 229-238.

(42) Chiang, H.; Bhan, A. Catalytic consequences of hydroxyl group location on the rate and mechanism of parallel dehydration reactions of ethanol over acidic zeolites. J. Catal. 2010, 271 (2), 251-261.

(43) Williams, C.; Makarova, M. A.; Malysheva, L. V.; Paukshtis, E. A.; Zamaraev, K. I.; Thomas, J. M. Mechanistic studies of the catalytic dehydration of isobutyl alcohol on NaH-ZSM-5. J. Chem. Soc. Faraday Trans. 1990, 86 (20), 3473-3485.

(44) Liu, D.; Bhan, A.; Tsapatsis, M.; Al Hashimi, S. Catalytic behavior of Brønsted acid sites in MWW and MFI zeolites with dual meso- and microporosity. ACS Catal. 2011, 1 (1), 7-17.

(45) Kumar, G.; Liu, D.; Xu, D.; Tsapatsis, M.; Dauenhauer, P. J.; Dehydra-Decyclization of 2Methyltetrahydrofuran to Pentadienes on BoronContaining Zeolites. Green Chem. Advanced article 2020 (DOI: 10.1039/D0GC00136H).

(46) Gounder, R.; Iglesia, E. The catalytic diversity of zeolites: confinement and solvation effects within voids of molecular dimensions. Chem. Commun. 2013, 49 (34), 3491. 
(47) Kumbhalkar, M. D.; Buchanan, J. S.; Huber, G. W.; Dumesic, J. A. Ring Opening of Biomass-derived Cyclic Ethers to Dienes over Silica/Alumina. ACS Cat. 2017, 8, 5248-5256.

(48) Scott, S. L. A Matter of Life(time) and Death. ACS Catal. 2018, 8, 8597-8599.

(49) C\&EN article https://cen.acs.org/articles/91/i5/Coping-HeliumShortage.html (accessed 3 May 2020).

(50) Alexopoulos, K.; John, M.; Van Der Borght, K.; Galvita, V.; Reyniers, M. F.; Marin, G. B. DFT-based microkinetic modeling of ethanol dehydration in $\mathrm{H}-$ ZSM-5. J. Catal. 2016, 339, 173-185.

(51) Makarova, M. A.; Paukshtis, E. A.; Thomas, J. M.; Williams, C.; Zamaraev, K. I, Dehydration of nButanol on Zeolite H-ZSM-5 and Amorphous Aluminosilicate: Detailed Mechanistic Study and the Effect of Pore Confinement, J. Catal. 1994, 149, 3651.

(52) Phillips, C. B.; Datta, R. Production of ethylene from hydrous ethanol on H-ZSM-5 under mild conditions, Ind. Eng. Chem. Res. 1997, 36 (11), 44664475.

(53) Moser, W. R.; Thompson, R. W.; Chiang, C. C.; Tong, H. Silicon-rich H-ZSM-5 catalyzed conversion of aqueous ethanol to ethylene, J. Catal. 1989, 117 (1), 19-32.

(54) John, M.; Alexopoulos, K.; Reyniers, M. F.; Marin, G. B., First-Principles Kinetic Study on the Effect of the Zeolite Framework on 1-Butanol Dehydration, ACS Catal. 2016, 6 (7), 4081-4094.

(55) John, M.; Alexopoulos, K.; Reyniers, M.; Marin, G. B. Reaction path analysis for 1-butanol dehydration in H-ZSM-5 zeolite: $\mathrm{Ab}$ initio and microkinetic modeling, J. Catal. 2015, 330, 28-45. 